\title{
La manutenzione dei pozzi per acqua: cause della perdita di efficienza dei pozzi e principali tecniche di intervento.
}

\author{
Nicola Cempini \\ Ingegnerie Toscane srl. \\ n.cempini@ingegnerietoscane.net
}

Fin dall'inizio della vita operativa i pozzi per acqua subiscono un processo di deterioramento, in particolare di occlusione dei filtri, spesso del dreno, che ne causa la perdita di efficienza idraulica.

Questo processo di deterioramento, che interessa anche la tubazione cieca, ha una serie di cause quali occlusioni chimiche, fisiche, biologiche; corrosione; lesioni da correnti vaganti; rotture (spesso delle saldature).

Il well blocking è un processo che talvolta avviene in pochi anni ed è purtroppo 'autoalimentante' nel senso che più il filtro è intasato e più tende ad intasarsi: il restringimento della sezione filtrante causa infatti un aumento della velocità dell'acqua che perciò è capace di mobilizzare anche le particelle più fini dell'acquifero. L'aumento della turbolenza determina inoltre la maggiore degassazione dell'acqua: i gas (come la $\mathrm{CO}_{2}$ ) se ne vanno favorendo la precipitazione dei minerali, soprattutto ossidi di ferro e manganese e carbonato di calcio.

Altro motivo importante di well blocking è la proliferazione batterica. Sulla superficie dei filtri, ma anche sulla tubazione cieca, si può formare un biofilm.

Il biofilm è una aggregazione complessa di microorganismi che consiste in una formidabile strategia di sopravvivenza. I batteri secernono una matrice comune, costituita fondamentalmente da esopolimeri, soprattutto polisaccaridi, che non di rado sfrutta la presenza di incrostazioni minerali formando una combinazione di depositi minerali e biologici; ogni batterio può produrre esopolimeri da 30 a 100 volte il suo peso.

Questa matrice forma un brodo di coltura autoprodotto che ha la capacità di aderire con forza al substrato, soprattutto metallico. La formazione del biofilm inizia con l'ancoraggio dei microorganismi presenti nell'acqua (free swimming) alla superficie del filtro; le prime adesioni avvengono attraverso le deboli forze di Van der Waals. Il biofilm consente ai batteri di essere molto più resistenti agli agenti ostili rispetto ai microorganismi fluttuanti.

Un singolo biofilm può ospitare diverse specie di batteri, sia aerobici (soprattutto microareofili) che anaerobici. I primi si trovano più vicini alla superficie e al flusso dell'acqua mentre i secondo si piazzano nelle zone più interne e più vicini al substrato ed è proprio qui che le condizioni redox favoriscono l'innesco della biocorrosione.

Il biofilm può rimanere una massa sostanzialmente organi$\mathrm{ca}$, ma più frequentemente va a costituire il substrato ideale per la precipitazione di aggregati minerali diventando una barriera potente allo scorrimento dell'acqua.

Le lesioni da correnti vaganti (correnti disperse nel terreno da tutti gli impianti elettrici con presa a terra: linee di trazio- ne elettrica con ritorno su rotaia; impianti radiofonici e telefonici, ecc.) compromettono gravemente la funzionalità del pozzo. Il fenomeno produce fori rotondeggianti che raggiungono in breve dimensioni di alcuni $\mathrm{cm}$ di diametro consentendo la fuoriuscita del dreno dalla propria sede.

Queste correnti incontrando nel loro percorso un materiale metallico vi penetrano a causa della minore resistività di questo rispetto al terreno e quindi ne fuoriescono; sfruttano il metallo interrato come corsia preferenziale nel loro viaggio di ritorno alla sottostazione. Il punto in cui si verifica la lesione è il punto di uscita della corrente.

Le lesioni da corrosione galvanica si verificano qualora due metalli o parti dello stesso metallo a diverso potenziale vengono posti in opera in contatto, in presenza di un elettrolita quale l'acqua di falda: si forma una cella galvanica in corto circuito. Sulla superficie dei manufatti in acciaio, a causa delle lavorazioni, ci sono sempre zone a potenziale redox diverso a causa di differenze fisico chimiche anche minime nella composizione del metallo. Differenze di potenziale possono essere dovute all'azione di alcuni tipi di batteri, soprattutto i riduttori di solfati, che col loro metabolismo inducono la formazione di aree a potenziale diverso innescando la biocorrosione.

Sovente capita di osservare rotture e/o corrosione in corrispondenza delle saldature. Queste in effetti rappresentano punti critici della stringa di completamento se non eseguite a regola d'arte. Questo problema riguarda soprattutto l'acciaio inox; quello di più frequente utilizzo è l'AISI 304. L'alta temperatura che si sviluppa durante la saldatura può determinare il decadimento della struttura da austenitica a ferritica (Nicodemi, 2000 e 2003). È fondamentale che la saldatura dell'inox sia eseguita con metodo TIG con elettrodo infusibile. Sarebbe inoltre opportuno procedere al decapaggio e alla passivazione della saldatura, ma questa è una operazione non semplicissima da eseguire in cantiere e soprattutto impossibile da eseguire sul lato interno delle barre. Il professionista deve diagnosticare con esattezza lo stato del pozzo; il primo step consiste nel reperire dal gestore tutte le informazioni utili sulla vita operativa: tipo di pompa installata, portata iniziale e attuale, schema di completamento e stratigrafia, problematiche osservate dagli operatori in particolar modo la diminuzione di portata nel tempo ed eventuali variazioni significative del livello statico e dinamico. È altresì importante verificare la presenza nell'acqua di materiale in sospensione e/o tracce biologiche (come ad esempio i filamenti dei ferrobatteri); in caso positivo è opportuno eseguire l'analisi su due campioni: uno dopo uno stop significativo del pompaggio (12-24 ore), l'altro dopo almeno 5 ore di pompaggio. Il biofilm infatti reagisce 
ai cambiamenti del regime del flusso dell'acqua: quando questo è forte la biopellicola si assottiglia, si contrae impedendo ai batteri di uscirne. Quando invece il pozzo è inattivo e il flusso è naturale, il biofilm assorbe acqua, si espande e rilascia molti più batteri nel pozzo. Leggendo le analisi si deve tenere conto del fatto che il rapporto numerico tra batteri free swimming e batteri del biofilm è di $1: 10^{6}$. Per ogni batterio presente nell'acqua ce ne sono ben 1.000.000 attaccati alle pareti (Schneiders, 2003). I batteri da ricercare nelle analisi sono sostanzialmente di tre tipi: coliformi (slime formers); riduttori di solfati (soprattutto Desulfovibrio); ferrobatteri: l'ospite più frequente nei pozzi per acqua è la Gallionella ferruginea (Hallbeck L. \& Pedersen K., 1990).

Il secondo step è quello di eseguire una video ispezione: si tratta di una operazione che consente di rendersi conto direttamente dello stato del pozzo e di stendere un protocollo di intervento specifico efficace. La video ispezione dovrebbe essere eseguita di regola dopo 24 ore dall'estrazione della pompa, in modo da non avere a che fare con un acqua eccessivamente torbida. Le operazioni di espianto pompa infatti causano l'intorbidamento dell'acqua. Si può contrarre questo tempo se le esigenze operative lo richiedono, ma è bene non scendere sotto le 6 ore. L'esecuzione di un log televisivo non è una operazione banale: la telecamera non deve scendere troppo velocemente, bisogna fermarsi e insistere nelle zone delle saldature, in presenza di fenomeni di corrosione, di incrostazioni, ecc.

Una volta eseguita e analizzata la video ispezione, reperite le informazioni fondamentali, il water well professional è in grado di stabilire le cause della perdita di efficienza dell'opera e indicare le possibili soluzioni.

I metodi più comuni per l'esecuzione della manutenzione sono il pistonaggio, il jetting tool, l'bydropuls ${ }^{\circledR}$ e il brushing. A ognuno di questi metodi può essere abbinato o meno il trattamento chimico.

Quando si ha che fare con incrostazioni minerali o biologiche o entrambe, occorre innanzitutto procedere ad una pulizia meccanica. Dalla video ispezione si evincono le quote di intervento ovvero la posizione precisa alla quale bisogna pistonare oppure operare con un altro utensile. Bisogna prestare molta attenzione all'energia con cui si procede a questa operazione che va eseguita tenendo ben presente lo stato generale di conservazione del pozzo, in quanto si rischia di lesionare ancora di più il tubo o addirittura causarne il franamento.

Al termine della fase di pulizia meccanica bisogna rimuovere il detrito, generalmente col metodo dell'air lift. In alcune occasioni, pozzi recenti, gestiti correttamente e mantenuti regolarmente, la semplice operazione di pulizia meccanica può bastare per far tornare l'opera a livelli di efficienza soddisfacenti. Spesso invece occorre continuare la manutenzione con un nuovo ciclo di pulizia meccanica abbinata a lavaggio chimico ovvero all'uso di uno o più prodotti specifici per il trattamento delle problematiche riscontrate. Sul mercato sono presenti prodotti per la rimozione di incrostazioni minerali (ferro, manganese, carbonati), per la rimozione del biofilm, per la rimozione delle particelle fini, per la disinfezione del pozzo. Alcuni di questi prodotti possono essere utilizzati in- sieme, secondo le indicazioni del produttore, per il trattamento incrostazioni miste batterico-minerali.

Non sempre è conveniente procedere alla manutenzione. Le tecniche attuali consentono buone capacità di recupero nella grande maggioranza dei casi ma l'opera può presentarsi in condizioni tali da rendere antieconomica la manutenzione. Il professionista deve quindi indicare al committente le possibili soluzioni: ritubaggio o abbandono e esecuzione di una nuova opera.

Il ritubaggio di un pozzo è la posa in opera di una nuova stringa di completamento (generalmente in materiale termoplastico ma anche in accaio) all'interno di un pozzo lesionato al punto tale da impedire la fruibilità dell'opera.

Il ritubaggio deve essere preceduto o seguito da una manutenzione che assicuri la maggiore pulizia possibile della vecchia tubazione; in effetti ritubare un pozzo intasato non avrebbe molto senso non consentendo alcun recupero. Tuttavia una pulizia preventiva non sempre è possibile; spesso infatti i pozzi da ritubare sono in condizioni tali da escludere la possibilità di intervenire energicamente pena il franamento dell'opera che impedirebbe qualsiasi recupero. In questi casi è possibile procedere alla pulizia successivamente alla posa della nuova stringa; si dovrà procedere soprattutto sul versante del lavaggio chimico.

Quando la manutenzione e il recupero non sono più possibili o antieconomici, si può procedere all'esecuzione di un nuovo pozzo o utilizzare una delle interessanti tecniche di estrazione delle aste e ricostruzione oggi disponibili.

\section{BIBLIOGRAFIA}

Hallbeck L. \& Pedersen K.(1990). Culture parameters regulating stalk formation and growth rate of Gallionella ferruginea. Journal of general microbiology 136, 1675-1680.

Schneiders J.H. (2003) Chemical cleaning, disinfection \& decontamination of water wells. Editore Johnson Screens.

Nicodemi W. (2000). Acciai e leghe non ferrose. Editore Zanichelli.

Nicodemi W. (2003). Introduzione agli acciai inossidabili. Editore AIM. 